Internist $2010 \cdot 52: 889-893$

DOI 10.1007/s00108-010-2742-y

Online publiziert: 28. Oktober 2010

(c) Springer-Verlag 2010

\section{Rubrikherausgeber}

K. Werdan, Halle (Saale)

D. Vuichard ${ }^{1} \cdot$ M.J. Zellweger ${ }^{2} \cdot$ M. Altwegg $^{3} \cdot$ R. Frei ${ }^{4} \cdot$ M. Weisser ${ }^{1}$

${ }^{1}$ Klinik für Infektiologie \& Spitalhygiene, Universitätsspital Basel

${ }^{2}$ Kardiologische Klinik, Universitätsspital Basel

${ }^{3}$ Bio-Analytica AG, Luzern

${ }^{4}$ Abteilung für Klinische Mikrobiologie, Universitätsspital Basel

\title{
44-jähriger Patient mit kulturnegativer, purulenter Perikarditis
}

\section{Technische Untersuchungen}

In der Laboranalyse imponierten eine Leukozytose von $13,4 \times 10^{9} / 1$ (normal: $\left.3,5-10 \times 10^{9} / 1\right)$ mit $84 \%$ neutrophilen Granulozyten und ein CRP von $339 \mathrm{mg} / \mathrm{l}$ (normal: $<10,0)$. Elektrolyte, Transaminasen und Kreatinin sowie Kreatinkinase, Troponin und Myoglobin waren normwertig.

Im Thoraxröntgenbild zeigte sich eine deutlich verbreiterte Herzsilhouette. In der Elektrokardiographie (EKG) fielen diffuse ST-Hebungen und eine PQ-Senkung in Ableitung II auf (• Abb. 1). Die transthorakale Echokardiographie bestätigte einen deutlichen Perikarderguss mit beginnender hämodynamischer Relevanz (- Abb. 2). Bei der unmittelbar durchgeführten Perikardiozentese wurden $500 \mathrm{ml}$ einer trüben Flüssigkeit drainiert. Die mikroskopische Differenzierung ergab eine Zellzahl von $28 \times 10^{9} / 1\left(<1 \times 10^{9} / 1\right)$ mit $98 \%$ polymorphkernigen Zellen. Die LDHAktivität betrug $2316 \mathrm{U} / \mathrm{l}$ (135-225), das Gesamtprotein 6o g/l (62-80), die Glukose $4,2 \mathrm{mmol} / \mathrm{l}(3,8-6,1)$ und der Hämatokrit o,o 1/l. Die Gramfärbung und die Auramin-Rhodamin-Färbung für säurefeste Stäbchen waren negativ. Die Zytologie ergab keinen Hinweis auf Malignität.

\section{Verdachtsdiagnose \\ - Eitriger Perikarderguss, beginnend hämodynamisch relevant, am ehesten bakterieller Genese, bei Vor- behandlung mit Antibiotika bislang ohne Erregerdiagnose}

\section{Weiterführende Untersuchungen}

Zwei Tage nach Krankenhausaufnahme wurde zusätzlich $650 \mathrm{ml}$ Pleuraflüssigkeit drainiert. Das Exsudat (LDH Pleura/ in Kontakt mit Tieren. Eine mögliche Tuberkulose- oder HIV-Exposition verneinte er. Ebenso wenig fanden sich anamnestisch Indizien für eine zugrunde liegende rheumatologische Erkrankung.

\section{Befunde}

\section{Körperlicher Untersuchungsbefund}

Der Patient war afebril $\left(37,2^{\circ} \mathrm{C}\right)$, tachykard $(112 / \mathrm{min})$ und normoton. Auffällig waren die gestauten Halsvenen sowie leise Herztöne. Lungen und Abdomen waren unauffällig. Haut und Schleimhäute zeigten keine Veränderungen. Der Patient hatte keinen Meningismus. Kraft, Sensibilität und Muskeleigenreflexe waren normal.

\section{Tab. 1 Indikationen für eine Breitspektrum-Polymerasekettenreaktion (PCR)}

- Nachweis von Erregern, die mit den konventionellen Methoden nicht oder nur schwer anzüchtbar sind, z. B. Tropheryma whipplei, Bartonella spp., Coxiella burnetii, Mycoplasma pneumoniae

- Nachweis von Bakterien, die wegen Vorbehandlung mit Antibiotika nicht mehr in der Kultur wachsen

\section{Voraussetzung:}

- Material aus primär steriler Lokalisation

- Sterile Entnahmetechnik

- Vermutete Monoinfektion

\section{Grenzen der Methode:}

- Eingeschränkte Sensitivität im Vergleich mit speziesspezifischen PCR-Verfahren

- Vorliegen einer Mischsequenz (Kontamination, Mischinfektion)

- Bei Mischinfektionen wird ggf. nur der in der Keimzahl dominierende Erreger nachgewiesen (Fehlinterpretation: Monoinfektion)

- Keine Resistenzprüfung 


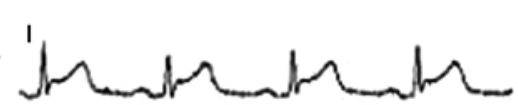

II

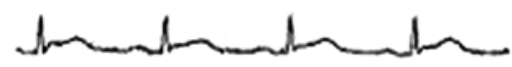

se

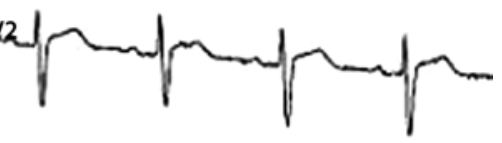

III

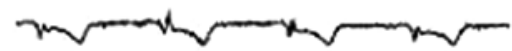

aVR

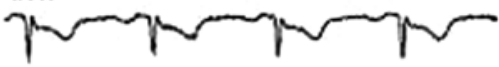

aVL
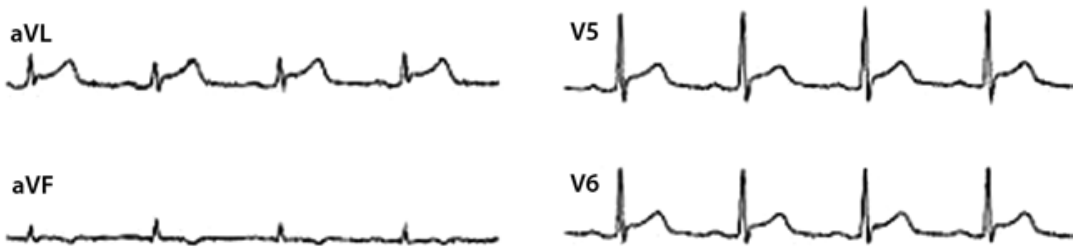

$25 \mathrm{~mm} / \mathrm{s} 10.0 \mathrm{~mm} / \mathrm{mV}$

Abb. $1 \Delta$ Diffuse ST-Hebungen und PQ-Senkungen gut sichtbar in Ableitung II

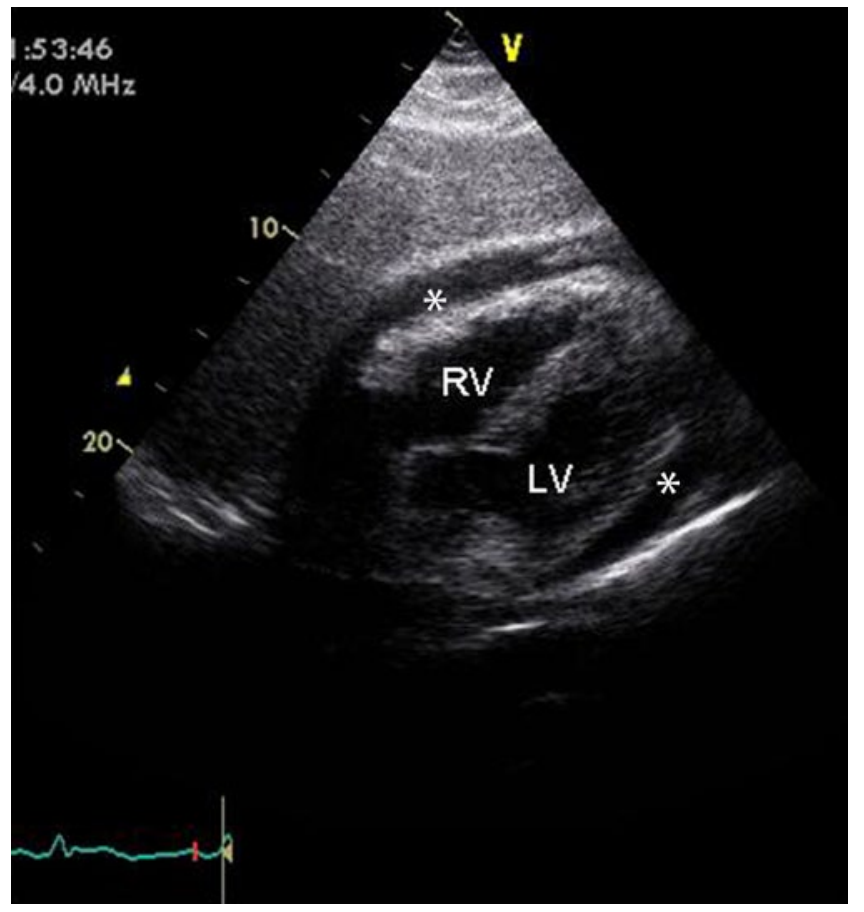

Abb. $2<$ Transthorakale Echokardiographie. $R V$ : rechter Ventrikel, LV: linker Ventrikel, * zirkulärer Perikarderguss

Serum >0,6; Gesamtprotein 33 g/l, Zell-

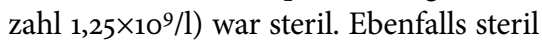
im Verlauf waren die mehrfach, allerdings stets unter antimikrobieller Therapie, entnommenen Blutkulturen. Die Kulturen und die spezifische Polymerasekettenreaktion (PCR) für Chlamydophila pneumoniae und Mycobacterium-tuberculosis-Komplex aus dem Perikardaspirat wie auch ein
Antigentest für Streptococcus pneumoniae aus der Perikardflüssigkeit und dem Urin waren negativ. Ein kombinierter HIV-Test (Antikörper + p24-Antigen) war nicht reaktiv. Borrelien-IgG und -IgM im Serum waren negativ. Rheumafaktoren und antineutrophile zytoplasmatische Antikörper (ANCA) waren normwertig, der Titer der antinukleären Antikörper (ANA) war mit
1:320 (1:40) und im Verlauf 1:640 erhöht. Serumprotein $\mathrm{CH}_{50}$ und Antistreptolysin lagen im Normbereich.

Eine eubakterielle PCR-Amplifikation aus dem Perikardpunktat zeigte schließlich DNA-Sequenzen, die denjenigen von Neisseria meningitidis, $N$. cinerea und $N$. polysaccharea entsprachen und eindeutige Mismatches mit anderen Neisseria spp. aufwiesen. Ein Latexagglutinationstest für Meningokokkenantigene aus dem Pleurapunktat war positiv für N. meningitidis der Serogruppe C.

\section{Diagnose \\ - Primär eitrige Perikarditis mit Menin- gokokken der Serogruppe $C$}

\section{Therapie und Verlauf}

Die empirische Therapie mit Amoxicillin/Clavulansäure $3 \times 2,2 \mathrm{~g}$ i.v. wurde nach 3 Tagen aufgrund von ausgeprägter Nausea durch Cefazolin $3 \times 2$ g i.v. ersetzt. Nach 2-wöchiger parenteraler Antibiotikatherapie mit Regredienz der Beschwerden und Entzündungsparameter wurde der Patient nach Hause entlassen. Eine Magnetresonanztomographie des Herzens zeigte lediglich geringe Perikardverdickungen und keine pathologische Kontrastmittelanreicherung im Myokard als Hinweis für eine Perikarditis constrictiva oder entzündliche Myokardbeteiligung (• Abb. 3). Zwei Monate später war der Patient symptomfrei und die Echokardiographie unauffällig.

Drei Monate nach Entlassung präsentierte sich der Patient mit Fieber und linksseitigen Thoraxschmerzen beim Husten mit Ausstrahlung in die linke Schulter. Bis auf die erhöhte Temperatur von $38,6^{\circ} \mathrm{C}$ war die klinische Untersuchung unauffällig. Das EKG zeigte eine PQ-Senkung in Ableitung I (• Abb.4), das Labor eine Leukozytose von $12 \times 10^{9} / 1$ und ein CRP von $85 \mathrm{mg} / \mathrm{l}$, bei normwertigen kardialen Markern. Die transthorakale Echokardiographie ergab einen kleinen, nicht punktionswürdigen Perikarderguss und nebenbefundlich moderate Pleuraergüsse beidseits. Wir postulierten eine immunreaktive Genese und begannen mit nichtsteroidalen Antirheumatika. Bei Fieberpersistenz und septischem Zustandsbild wurde nach 2 Tagen Ceftriaxon $1 \times 2 \mathrm{~g}$ 
täglich i.v. initiiert. Blutkulturen und ein Pleurapunktat blieben steril. Ein Immunglobulinmangel und ein Komplementdefekt wurden ausgeschlossen. In der Verlaufsechokardiographie war der Perikarderguss vollständig regredient. Ceftriaxon wurde für 14 Tage verabreicht. In den Verlaufskontrollen war der Patient stets beschwerdefrei.

\section{Abschließende Diagnose \\ - Primär eitrige Perikarditis mit Neisse- ria meningitidis der Serogruppe $\mathrm{C}$ mit Verdacht auf Infektrezidiv, differen- zialdiagnostisch reaktiver Erguss}

\section{Diskussion}

\section{Purulente Perikarditis}

Im Antibiotikazeitalter ist die purulente Perikarditis selten geworden. Insbesondere durch den raschen Einsatz von Antibiotika sieht man kaum mehr die Perikarditis im Rahmen einer Pneumonie mit Streptococcus pneumoniae. Heutzutage tritt die purulente Perikarditis häufiger nach chirurgischen Eingriffen, einem penetrierenden Trauma oder bei Immunsuppression auf.

\section{Eine mikrobiologische Diagnostik sollte vor Beginn der Antibiotikatherapie durchgeführt werden}

Vorherrschende Erreger sind grampositive Bakterien. Anaerobier und gramnegative Stäbchen kommen vorwiegend bei einer Perikarditis im Rahmen einer hämatogenen Streuung von einem abdominellen oder dentogenen Infektfokus vor. In Endemiegebieten und bei Patienten mit AIDS ist eine durch Mycobacterium tuberculosis verursachte Perikarditis häufig. Eine mikro- oder makroskopisch purulente Perikarditis muss nicht infektiös bedingt sein. Diverse systemische Erkrankungen wie beispielsweise ein Lupus erythematodes oder Neoplasien können ein entzündliches Exsudat mit erhöhter Zellzahl aufweisen [8]. Im Gegensatz dazu ist die Perikardflüssigkeit beispielsweise bei Mykoplasmen oder Viren selten purulent [7].

Eine mikrobiologische Diagnostik in dieser Situation ist zwingend. Hierzu ge- hören in erster Linie Blutkulturen. Wann immer möglich, sollte die Perikardiozentese oder Perikardiotomie vor dem Beginn der Antibiotikatherapie durchgeführt werden. Diese korrekte diagnostische Vorgehensweise wird in der Praxis leider allzu oft nicht durchgesetzt und führt zusammen mit dem raschen Einsatz von breit wirksamen Antibiotika zur erschwerten Diagnosestellung.

\section{Meningokokkenperikarditis}

Eine Perikarditis durch N. meningitidis ist selten. Typischerweise präsentiert sie sich mit Thoraxschmerzen (81\%), Fieber (70\%) und Dyspnoe (50\%). Verschiedene pathogenetische Mechanismen liegen zugrunde. So wird die primäre Perikarditis von einer Perikarditis als lokale Manifestation eines disseminierten Infekts und einer reaktiven (autoimmunen) Perikarditis unterschieden [3]. Am häufigsten ist die Perikarditis Komplikation einer Meningokokkenmeningitis oder einer fulminanten Sepsis. Die Entzündung des Perikards ist Folge der direkten Pathogeninvasion im Rahmen der Bakteriämie. Bei der primären, isolierten Perikarditis finden sich klinisch keine Hinweise für eine Meningitis oder Sepsis. Ursprung ist entweder eine Bakteriämie oder ein Infekt des unteren Respirationstrakts im Sinne einer per continuitatem entstandenen Infektion.

Die meisten Fälle von primärer Meningokokkenperikarditis wurden mit Stämmen der Serogruppe C beschrieben. Ältere Personen sind häufiger betroffen als Kinder, und der hämodynamisch relevante Perikarderguss respektive die Herztamponade sind viel häufiger als beim disseminierten Infekt [6].

Bei der reaktiven Form entwickelt sich im späteren Verlauf einer Meningokokkeninfektion und nach einer durchaus adäquaten antibiotischen Behandlung ein progredienter Perikarderguss, welcher meist serös und steril ist, ähnlich einer postviralen Perikarditis. Häufig finden sich gleichzeitig auch andere aseptische Entzündungsherde wie eine Pleuritis oder Arthritis. Zirkulierende Immunkomplexe werden als Ursache vermutet [3].

Warum es bei unserem Patienten nach 3 Monaten zu einer neuerlichen Episode
Internist 2010 - 52:889-893

DOI 10.1007/s00108-010-2742-y

() Springer-Verlag 2010

D. Vuichard · M.J. Zellweger · M. Altwegg • R. Frei $\cdot$ M. Weisser 44-jähriger Patient mit kulturnegativer, purulenter Perikarditis

Zusammenfassung

Wir berichten über einen Patienten mit purulenter Perikarditis und drohender Perikardtamponade, verursacht durch Neisseria meningitidis. Bei negativen Kulturen aus Blut und Perikardpunktat, wahrscheinlich bedingt durch die Vorbehandlung mit Antibiotika, konnten wir mittels Breitspektrumpolymerasekettenreaktion aus dem Perikardpunktat und einem Latexagglutinationstest aus Pleurapunktat Meningokokken der Serogruppe $\mathrm{C}$ nachweisen. Eine Meningokokkenperikarditis ohne Meningitis ist selten. Diagnostische Methoden auf nicht-kultureller Basis spielen bei Patienten, die bereits mit Antibiotika vorbehandelt sind, eine wichtige Rolle.

Schlüsselwörter

Perikarditis, eitrig · Meningokokken .

Neisseria meningitidis .

Polymerasekettenreaktion .

Antibiotikatherapie

\section{Culture-negative, purulent pericarditis}

Abstract

We report about a patient with purulent pericarditis due to Neisseria meningitidis pretreated with antibiotics. Clinical signs were suggestive of pericardial tamponade. Cultures from blood and pericardial aspirate remained negative. Broad-range polymerase chain reaction from pericardial fluid detected Neisseria $s p$. .. Latex agglutination assay from pleural fluid showed positive reaction with meningococcal antigen serogroup C. Meningococcal pericarditis without meningitis is a rare manifestation. Non-culture based diagnostic methods in patients with such severe infections and negative cultures play an important role.

Keywords

Pericarditis, purulent $\cdot$ Meningococci . Neisseria meningitidis · Polymerase chain reaction - Antibiotic therapy 


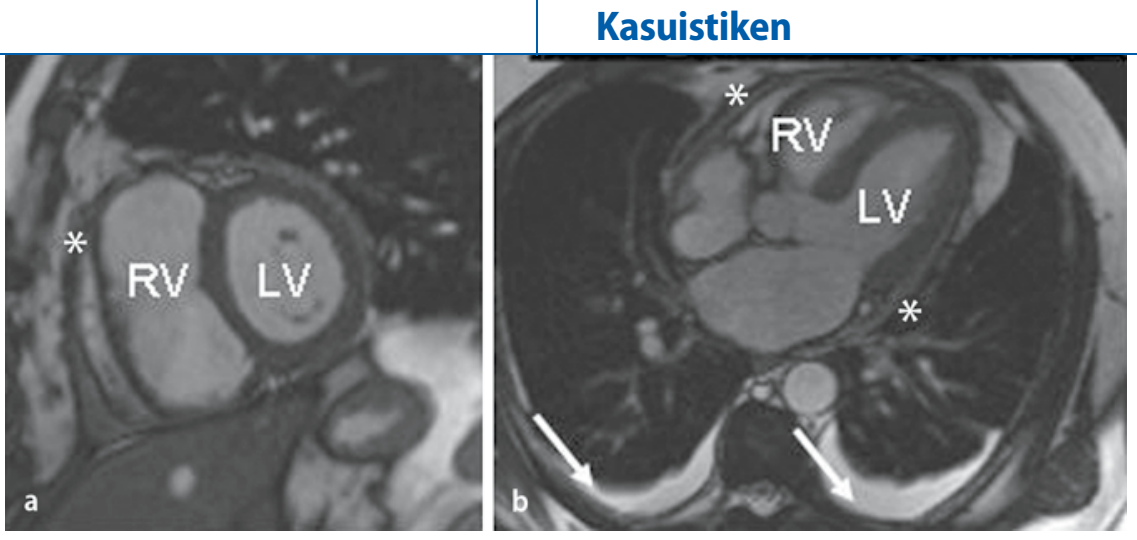

Abb. $3 \Delta$ Magnetresonanztomographie des Herzens: a Kurze Achse des Herzens, b 5-Kammerblick. RV: rechter Ventrikel, LV: linker Ventrikel, * verdickte Perikardabschnitte Pfeile: Pleuraerguss beidseits
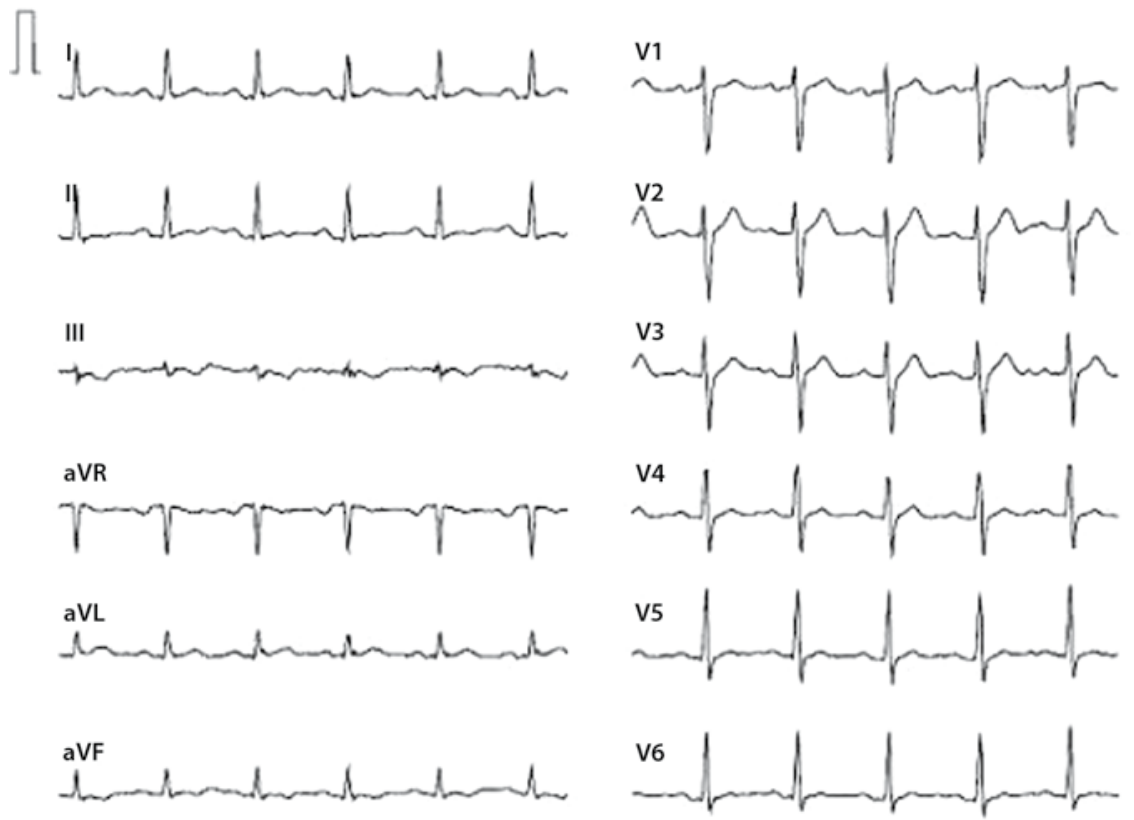

$25 \mathrm{~mm} / \mathrm{s} \quad 10.0 \mathrm{~mm} / \mathrm{mV}$

Abb. $4 \Delta$ PQ-Senkung gut sichtbar in Ableitung I

einer Serositis von Perikard und Pleura kam, bleibt letztendlich unklar. Eine Erregerdiagnose gelang nicht. Ob es sich tatsächlich um eine infektiöse Genese handelte, bleibt offen. Bei einem Komplementdefekt werden rezidivierende Infektionen mit Neisserien und ein bis zu 6ooo-fach erhöhtes Risiko für eine systemische Meningokokkeninfektion beobachtet [2]. Die Komplementaktivierung bei unserem Patient war normal. Für eine vermutete reaktive Perikarditis im Sinne einer Autoimmunerkrankung - wie oben beschrieben - fanden sich klinisch und laborchemisch keine Hinweise, und das prompte Ansprechen auf eine Antibiotikatherapie spricht dagegen.

\section{Diagnostik von \\ Meningokokkeninfektionen}

Die Diagnose einer Infektion mit N. meningitidis wird in der Regel anhand eines Direktpräparats oder von Kulturen aus diversen primär sterilen Materialien wie Blut, Liquor oder Gelenkflüssigkeit gestellt. Auf nicht-kultureller Basis existieren der Latexagglutinationstest gegen N.-meningitidis-Antigene aus Serum oder Liquor und molekulargenetische Nachweismethoden. Von den 13 Serogruppen weist der Latexagglutinationstest nur die häufigsten Kapselpolysaccharidantigene nach (A, C, Y und W135). Die Sensitivität variiert stark in Abhängigkeit von der angewandten Methode und der Keimmenge im zu untersuchenden Material $[4,5]$.
Bei einer Meningitis oder Sepsis und fehlendem Kulturnachweis aufgrund vorangegangener Antibiotikatherapie ist die Anwendung einer spezifischen PCR zur Diagnostik von Meningokokken-DNA aus Liquor, Blut oder Hautläsionen nützlich [9]. In unserem Fall konnten wir bezüglich der Anamnese und der Klinik auf keinen spezifischen Erreger schließen. Mittels einer eubakteriellen oder „Breitspektrum"- (16S rRNA) PCR und nachfolgender Sequenzierung des Amplikons gelang uns der Nachweis von Neisseria sp. Diese anderweitig beschriebene Methode erfasst praktisch jegliche bakterielle DNA und erlaubt häufig eine genaue Identifizierung des Erregers [1]. Die Sensitivität einer eubakteriellen PCR ist allerdings weitaus geringer als jene der erregerspezifischen PCR (• Tab. 1). Ein nachträglich durchgeführter Latexagglutinationstest aus Material vom Pleurapunktat war schließlich reaktiv für Meningokokkenantigen der Serogruppe $\mathrm{C}$ und somit beweisend.

Das Follow-up eines solchen Patienten ist wichtig im Hinblick auf die mögliche Komplikation einer Perikarditis constrictiva. Neben der transthorakalen Echokardiographie kann eine magnetresonanztomographische Diagnostik hinzugezogen werden. Diese ist hinsichtlich der Aussage über Perikardverdickung, Myokardcharakterisierung und Abgrenzung des Perikards gegenüber umliegenden Strukturen der Echokardiographie überlegen. Diese Methode ist wegen der optimalen Funktionsaufnahmen und Myokardcharakterisierung sowie der fehlenden Strahlenbelastung einer computertomographischen Untersuchung vorzuziehen.

\section{Fazit für die Praxis}

Die primäre Meningokokkenperikarditis ist insgesamt selten. Ein hämodynamisch relevanter Perikarderguss ist häufiger als beim disseminierten Infekt und bedarf einer raschen Intervention. Bei einer erschwerten Diagnosestellung durch eine antibiotische Vorbehandlung können nicht-kulturelle Nachweismethoden wie ein Antigentest oder PCR-Verfahren eine rasche Diagnose liefern und ausgedehnte weiterführende Untersuchungen vermeiden, welche in den meisten Fällen unergiebig sind. 


\section{Korrespondenzadresse}

\section{Dr. D. Vuichard}

Klinik für Infektiologie \& Spitalhygiene,

Universitätsspital Basel

Petersgraben 4, 4031 Basel

Schweiz

vuichardd@uhbs.ch

Dank. Wir danken Herrn Prof. Manuel Battegay für die kritische Durchsicht.

Interessenkonflikt. Der korrespondierende Autor gibt an, dass kein Interessenkonflikt besteht.

\section{Literatur}

1. Bosshard PP, Kronenberg A, Zbinden R et al (2003) Etiologic diagnosis of infective endocarditis by broad-range polymerase chain reaction: a 3-year experience. Clin Infect Dis 37:167-172
2. Densen $P(1989)$ Interaction of complement with Neisseria meningitidis and Neisseria gonorrhoeae. Clin Microbiol Rev 2 (Suppl):S11-S17

3. Finkelstein Y, Adler Y, Nussinovitch M et al (1997) A new classification for pericarditis associated with meningococcal infection. Eur J Pediatr 156:585588

4. Finlay FO, Witherow H, Rudd PT (1995) Latex agglutination testing in bacterial meningitis. Arch Dis Child 73:160-161

5. Gray SJ, Sobanski MA, Kaczmarski EB et al (1999) Ultrasound-enhanced latex immunoagglutination and PCR as complementary methods for non-culture-based confirmation of meningococcal disease. J Clin Microbiol 37:1797-1801

6. Hardy DJ, Bartholomew WR, Amsterdam D (1986) Pathophysiology of primary meningococcal pericarditis associated with Neisseria meningitidis group C. A case report and review of the literature. Diagn Microbiol Infect Dis 4:259-265

7. Kenney RT, Li JS, Clyde WA Jr et al (1993) Mycoplasmal pericarditis: evidence of invasive disease. Clin Infect Dis 17 (Suppl 1):S58-62
8. Mandell BF (1987) Cardiovascular involvement in systemic lupus erythematosus. Semin Arthritis Rheum 17:126-141

9. Taha MK (2000) Simultaneous approach for nonculture PCR-based identification and serogroup prediction of Neisseria meningitidis. J Clin Microbiol 38:855-857

\section{Hier steht eine Anzeige.}

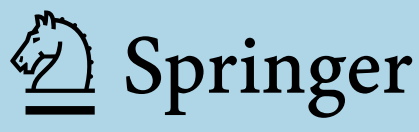

